MATHEMATICS OF COMPUTATION

Volume 70, Number 236, Pages 1543-1547

S 0025-5718(00)01262-X

Article electronically published on April 19, 2000

\title{
TENSOR PRODUCT GAUSS-LOBATTO POINTS ARE FEKETE POINTS FOR THE CUBE
}

\author{
L. BOS, M. A. TAYLOR, AND B. A. WINGATE
}

\begin{abstract}
Tensor products of Gauss-Lobatto quadrature points are frequently used as collocation points in spectral element methods. Unfortunately, it is not known if Gauss-Lobatto points exist in non-tensor-product domains like the simplex. In this work, we show that the $n$-dimensional tensor-product of Gauss-Lobatto quadrature points are also Fekete points. This suggests a way to generalize spectral methods based on Gauss-Lobatto points to nontensor-product domains, since Fekete points are known to exist and have been computed in the triangle and tetrahedron. In one dimension this result was proved by Fejér in 1932, but the extension to higher dimensions in non-trivial.
\end{abstract}

\section{INTRODUCTION}

The spectral finite element method 9 is a spectrally accurate algorithm for solving differential equations on unstructured grids. Typically the computational domain is broken into quadrilateral elements. Within each of these elements all variables are approximated by high degree polynomial expansions. The discrete equations are then derived using an integral form of the equations to be solved. When used with conforming elements and a clever choice of test functions and collocation points, the resulting mass matrix is diagonal [8].

The diagonal-mass-matrix spectral element method is only available with conforming quadrilateral grids. This is because the method relies on the existence of high order quadrature formulas which use the same number of collocation points as the number of basis functions. For the square, a tensor-product of Gauss-Lobatto quadrature points is used. One drawback of this method is that conforming quadrilateral grids can be quite complicated to generate, and the grids are rarely as uniform as triangulations. Thus there has been much work on on extending the spectral element method to triangular elements. The obvious approach would be to use Gauss-Lobatto quadrature points in the triangle. However, it is not known if such points exist for the triangle and so many other techniques have been developed [3, 10, 13, 2, 5, 12, 7.

The intent of this paper is to present a mathematical result related to the Fekete point generalization of the spectral element method proposed in [12, 7]. Fekete points are suggested as a way to generalize the Gauss-Lobatto quadrature points to non-tensor-product domains based on the following reasons:

Received by the editor November 10, 1999.

2000 Mathematics Subject Classification. Primary 41A10, 65D32, 65M60, 65M70.

Key words and phrases. Fekete Gauss Lobatto quadrature, spectral element methods.

(C)2000 American Mathematical Society 
1. On the $[-1,1]$ interval in the $1-\mathrm{D}$ case, Fekete points are the Gauss-Lobatto points [4].

2. On the square, Fekete points have been conjectured to be the tensor-product of the Gauss-Lobatto points. Thus the conventional spectral element method can also be considered a Fekete point method.

3. Under suitable assumptions, one can show that the Fekete points along each edge of the triangle are the Gauss-Lobatto points [1. This has been verified numerically up to degree $N=19$ [11]. Thus the Fekete points provide a natural coupling between triangular and quadrilateral elements.

4. Fekete points have near-optimal interpolation properties, and for degree $N>$ 10 they are the best interpolation points known for the triangle [11].

In this paper we present a proof of the conjecture in (2) above. In particular, we show that the $d$-dimensional tensor-product Gauss-Lobatto points are the unique Fekete points for the $d$-dimensional cube.

\section{Fekete-Gauss-Lobatto points for the interval}

On the interval $[-1,1]$ the Gauss quadrature points, $-1<b_{0}<b_{1}<\cdots<b_{n}<$ 1 , are such that the quadrature rule

$$
\int_{-1}^{1} f(x) d x \approx \sum_{i=0}^{n} w_{i} f\left(b_{i}\right)
$$

is exact for all polynomials of degree at most $2 n+1$. Here, the weights $w_{i}$ are given by

$$
w_{i}=\int_{-1}^{1} \ell_{i}(x) d x
$$

where $\ell_{i}(x)$ is the associated fundamental (or cardinal) Lagrange interpolating polynomial. As is well known, the end-points \pm 1 are not included in the Gauss quadrature points, and the Gauss-Lobatto points may be described as the "best" quadrature points that do include the end-points \pm 1 . Specifically, they are points $-1=a_{0}<a_{1}<\cdots<a_{n}=+1$ such that the quadrature rule

$$
\int_{-1}^{1} f(x) d x \approx \sum_{i=0}^{n} w_{i} f\left(a_{i}\right)
$$

is exact for all polynomials of degree at most $2 n-1$. (The weights $w_{i}$ are again given by the formula (2.1).)

In fact, they may be shown to be the zeros of the polynomial $\left(x^{2}-1\right) p_{n-1}^{\prime}(x)$, where $p_{k}$ is the $k$ th Legendre polynomial. Remarkably, as shown by Fejér in [4], these points are also the so-called Fekete points for the interval $[-1,1]$ defined to be those points in $[-1,1]$ for which the Vandermonde determinant

$$
V\left(x_{0}, \cdots, x_{n}\right):=\operatorname{det}\left(\begin{array}{ccccc}
1 & x_{0} & x_{0}^{2} & \cdots & x_{0}^{n} \\
1 & x_{1} & x_{1}^{2} & \cdots & x_{1}^{n} \\
\cdot & & & \cdots & \cdot \\
\cdot & & & \cdots & \cdot \\
1 & x_{n} & x_{n}^{2} & \cdots & x_{n}^{n}
\end{array}\right)
$$


is as large as possible. Since the fundamental Lagrange polynomials may be expressed in the form

$$
\ell_{i}(x)=\frac{V\left(a_{0}, \ldots, a_{i-1}, x, a_{i}, \ldots, a_{n}\right)}{V\left(a_{0}, \ldots, a_{n}\right)},
$$

we have for these Fekete-Gauss-Lobatto points the desirable property that

$$
\max _{-1 \leq x \leq 1}\left|\ell_{i}(x)\right|=1, \quad 0 \leq i \leq n .
$$

But Fejér showed that even the stronger inequality

$$
\max _{-1 \leq x \leq 1} \sum_{i=0}^{n} \ell_{i}^{2}(x)=1
$$

holds for these points. Later in this paper we will make use of the following simple remark.

Lemma 2.1. For the Fekete-Gauss-Lobatto points, the polynomial $\sum_{i=0}^{n} \ell_{i}^{2}(x)$ attains its maximum value of 1 at and only at the points $a_{i}$ themselves.

Proof. Let $F(x):=\sum_{i=0}^{n} \ell_{i}^{2}(x)$. Note that $F(x)$ is a polynomial of degree $2 n$. Now, by construction, $\ell_{i}\left(a_{j}\right)=\delta_{i j}$ and so it follows that

$$
F\left(a_{j}\right)=\sum_{i=0}^{n} \delta_{i j}^{2}=1, \quad 0 \leq j \leq n
$$

i.e., $F(x)$ does indeed attain its maximum value at the points $a_{i}$. But, at the interior points $-1<a_{1}<\cdots<a_{n-1}<1, F^{\prime}(x)$ must then be zero, and so $F(x)-1$ has a double zero at each of the interior $a_{i}$. This implies that $F$ attains the value 1 twice at each of the $n-1$ interior points, for a total of $2(n-1)$ times and once at each of the end-points for a grand total of $2(n-1)+2=2 n$ times. Since the degree of $F$ is $2 n$ it can attain the maximum value of 1 nowhere else in $[-1,1]$.

\section{Fekete-Gauss-Lobatto points for the cube}

Now consider tensor-product polynomial interpolation on the $d$-dimensional cube $[-1,1]^{d}$. A basis of tensor-product monomials may be described as follows. For $x=\left(x_{1}, \ldots, x_{d}\right) \in R^{d}$ and the multi-index $i=\left(i_{1}, \ldots, i_{d}\right)$, let

$$
x^{i}:=x_{1}^{i_{1}} x_{2}^{i_{2}} \cdots x_{d}^{i_{d}} .
$$

The tensor-product "degree" of $x^{i}$ is defined to be

$$
n=\operatorname{deg}\left(x^{i}\right)=|i|_{\infty}:=\max _{i \leq k \leq d} i_{k} .
$$

Then the $(n+1)^{d}$ monomials $m_{i}(x):=x^{i},|i|_{\infty} \leq n$ form a basis for the polynomials of tensor-product degree at most $n$.

Then, given a set of $(n+1)^{d}$ points

$$
\left\{A_{i}:|i|_{\infty} \leq n\right\} \subset[-1,1]^{d}
$$

and values $y_{i},|i|_{\infty} \leq n$, the interpolation problem is to find a linear combination $p(x)=\sum_{|i|_{\infty} \leq n} c_{i} x^{i}$ such that $p\left(A_{i}\right)=y_{i},|i|_{\infty} \leq n$. The associated Vandermonde matrix is the $(n+1)^{d} \times(n+1)^{d}$ matrix

$$
\left[m_{i}\left(A_{j}\right)\right]_{0 \leq|i|_{\infty},|j|_{\infty} \leq n}
$$


and the tensor-product Fekete points are those for which the determinant of this matrix is as large as possible.

Theorem 3.1. Suppose that $A_{i},|i|_{\infty} \leq n$, are the tensor products of the univariate Fekete-Gauss-Lobatto points $a_{i}$, i.e.,

$$
A_{i}:=\left(a_{i_{1}}, a_{i_{2}}, \ldots, a_{i_{d}}\right) .
$$

Then these are the unique tensor product Fekete points for the cube.

Proof. It is easy to see that the tensor-product Lagrange polynomials based on the $A_{i}$ are given by

$$
L_{i}(x)=\ell_{1}\left(x_{1}\right) \ell_{2}\left(x_{2}\right) \cdots \ell_{d}\left(x_{d}\right),
$$

where the $\ell_{i}$ are the univariate Lagrange polynomials based on the $a_{i}$. Further we may compute

$$
\sum_{|i|_{\infty} \leq n} L_{i}^{2}(x)=\prod_{k=1}^{d} \sum_{j=0}^{n} \ell_{j}^{2}\left(x_{j}\right)
$$

from which it follows that, for $x \in[-1,1]^{d}$,

$$
\sum_{|i|_{\infty} \leq n} L_{i}^{2}(x) \leq 1
$$

Now suppose that $B_{i},|i|_{\infty} \leq n$, is any other set of $(n+1)^{d}$ points in $[-1,1]^{d}$. Then, since the basis monomials are their own interpolants,

$$
m_{i}(x)=\sum_{|k|_{\infty} \leq n} m_{i}\left(A_{k}\right) L_{k}(x)
$$

and, in particular,

$$
m_{i}\left(B_{j}\right)=\sum_{|k|_{\infty} \leq n} m_{i}\left(A_{k}\right) L_{k}\left(B_{j}\right), \quad|j|_{\infty} \leq n .
$$

From this follows the Vandermonde matrix identity

$$
\left[m_{i}\left(B_{j}\right)\right]=\left[m_{i}\left(A_{j}\right)\right]\left[L_{i}\left(B_{j}\right)\right] .
$$

But now, Hadamard's inequality (see e.g., [6]) informs us that

$$
\left|\operatorname{det}\left(\left[L_{i}\left(B_{j}\right)\right]\right)\right| \leq \prod_{j} \sqrt{\sum_{i} L_{i}^{2}\left(B_{j}\right)},
$$

and hence, by (3.2), that

$$
\left|\operatorname{det}\left(\left[L_{i}\left(B_{j}\right)\right]\right)\right| \leq 1 .
$$

Applied to (3.3), this implies,

$$
\left|\operatorname{det}\left(\left[m_{i}\left(B_{j}\right)\right]\right)\right| \leq\left|\operatorname{det}\left(\left[m_{i}\left(A_{j}\right)\right]\right)\right|,
$$

or in words, that the Vandermonde determinant at any competing set of points $B_{i}$ is smaller in absolute value than the Vandermonde determinant for the tensor-product Fekete-Gauss-Lobatto points, i.e., these latter are indeed the tensor-product Fekete points!

As regards uniqueness, from Lemma 2.1 it follows that we have strict inequality in (3.2) for any $x$ not one of the tensor-product Fekete-Gauss-Lobatto points. Hence if even one of the competing points $B_{i}$ is not a tensor-product Fekete-Gauss-Lobatto 
point, we have strict inequality in (3.4), and so the $B_{i}$ could not also be Fekete points.

\section{REFERENCES}

[1] Bos, L., On certain configurations of points in $\mathbb{R}^{n}$ which are unisolvent for polynomial interpolation, J. Approx. Theory, 64, 271-280, 1991. MR 91m:41005

[2] Chen, Q., and I. Babuška, Approximate optimal points for polynomial interpolation of real functions in an interval and in a triangle, Comput. Methods Appl. Mech. Engrg., 128, 405417, 1995.

[3] Dubiner, M., Spectral methods on triangles and other domains, J. Sci. Comp., 6, 345-390, 1991. MR 92k:76061

[4] Fejér, L., Bestimmung derjenigen Abszissen eines Intervalles für welche die Quadratsumme der Grundfunktionen der Lagrangeschen Interpolation im Intervalle [-1,1] ein möglichst kleines Maximum besitzt, Ann. Scuola Norm. Sup. Pisa Sci. Fis. Mt. Ser. II, 1, 263-276, 1932.

[5] Hesthaven, J. S., and C. H. Teng, Stable spectral methods on tetrahedral elements, SIAM J. Sci. Comput., 1999, in press.

[6] Horn, R. A., and C. R. Johnson, Matrix analysis, Cambridge University Press, Cambridge, 1985. MR 87e:15001

[7] Komatitsch, D., et al., Wave propagation in 2-D elastic media using a spectral element method with triangles and quadrangles, submitted J. Comput. Acoust., 1999.

[8] Maday, Y. and A. T. Patera, Spectral element methods for the incompressible Navier-Stokes equations, in State of the Art Surveys in Computational Mechanics, edited by A. K. Noor, ASME, New York, 1988.

[9] Patera, A.T., A spectral element method for fluid dynamics: Laminar flow in a channel expansion, J. Comput. Phys., 54, 468-488, 1984.

[10] Sherwin, S. J., and G. E. Karniadakis, A triangular spectral element method: applications to the incompressible Navier-Stokes equations, Comput. Methods Appl. Mech. Engrg., 123, 189-229, 1995. MR 96b:76069

[11] Taylor, M. A., and B. A. Wingate, The Fekete collocation points for triangular spectral elements, SIAM J. Numer. Anal., 1998, submitted.

[12] Taylor, M. A., and B. A. Wingate, A generalized diagonal mass matrix spectral element method for non-quadrilateral elements, Appl. Num. Math., 1999, in press.

[13] Wingate, B. A., and J. P. Boyd, Spectral element methods on triangles for geophysical fluid dynamics problems, in Proceedings of the Third International Conference on Spectral and High-order Methods, edited by A. V. Ilin and L. R. Scott, pp. 305-314, Houston J. Mathematics, Houston, Texas, 1996.

Department of Mathematics and Statistics, University of Calgary, Calgary, Alberta CANADA

E-mail address: lpbos@math.ucalgary.ca

Los Alamos National Laboratory, Los Alamos, New Mexico

E-mail address: mt@lanl.gov

Los Alamos National Laboratory, Los Alamos, New Mexico

E-mail address: wingate@lanl.gov 